\title{
Temperature Dependent Solvation and Partitioning of Coumarin 152 in Phospholipid Membranes
}

Christine A. Gobrogge ${ }^{l}$, Victoria A. Kong ${ }^{2}$, and Robert A. Walker ${ }^{l *}$

AUTHOR INFORMATION

Department of Chemistry and Biochemistry, Montana State University, Bozeman, MT 59715.

\section{Supporting Information}

\section{Differential scanning calorimetry.}

A Texas Instruments Q2000 differential scanning calorimeter was used to determine the melting temperature of pure DMPC vesicle solution (20mM DMPC, rehydrated in 10mM PBS buffer, $\mathrm{pH}=7$ ). A second vesicle solution (20mM DMPC, rehydrated in PBS buffer, $10 \mathrm{mM}, \mathrm{pH}=7$, doped with $6 \mu \mathrm{M} \mathrm{C} 152)$ was used to verify the addition of $\mathrm{C} 152$ does not change the melting temperature of DMPC vesicle solutions. A pure buffer reference was used for both samples. DMPC vesicle solutions were equilibrated at $20^{\circ} \mathrm{C}$, after which the temperature was increased at a rate of $1^{\circ} \mathrm{C} \min ^{-1}$ from $20^{\circ} \mathrm{C}$ to $28^{\circ} \mathrm{C}$. Temperature sensitivity of the Q2000 DSC is $\pm 0.1^{\circ} \mathrm{C}$. Data were analyzed using TA Instruments Trios software. 


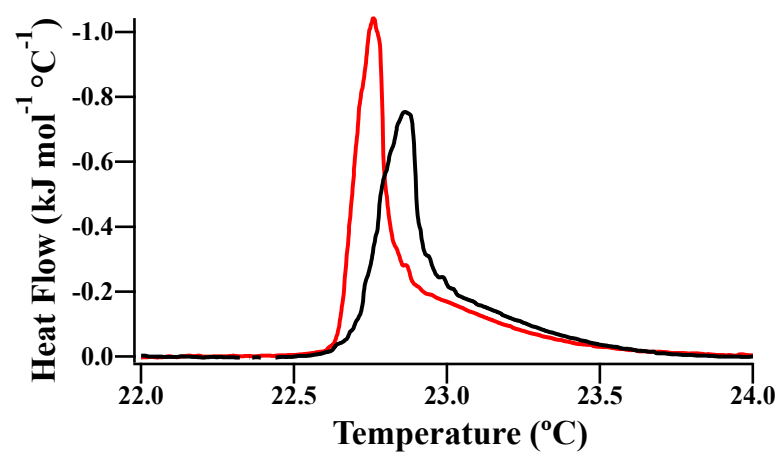

Figure S1. Differential Scanning Calorimetry traces of (red) 20mM DMPC vesicles and (black) 20mM DMPC vesicles doped with $6 \mu \mathrm{M}$ C152.

DMPC vesicles were found to have the same melting temperature (within error) with and without C152 in solution. 


\section{Quantum yield of C152 in PBS buffer.}

The quantum yield of C152 was measured in buffer over a temperature relevant to DMPC partitioning studies. The quantum yield was found to be $\sim 0.05$, as determined with absorption and integrated fluorescence of $\mathrm{C} 152$ in dilute buffer solutions. The quantum yield did to change with temperature relevant to studies described in this paper (Figure S2).

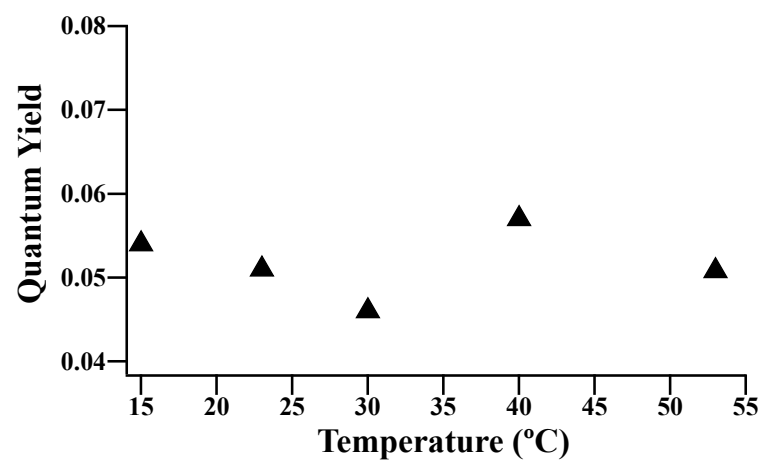

Figure S2. Quantum yields of C152 in bulk aqueous solution at varying temperatures.

In nonpolar solvents, the tertiary amine of $\mathrm{C} 152$ is $\mathrm{sp}^{3}$ hybridized, making the lone pair nonresonant with the rings' pi-system. When excited, C152 is promoted into an intramolecular charge transfer state, in which the amine has a similar geometry. This similarity between the ground state and excited state causes the radiative rate constant to be large, similarly making the quantum yield large and the fluorescent lifetime long. When C152 is excited in polar solvents, a charge separated twisted intramolecular charge transfer geometry is favored. Because the large difference in geometry between the ground and fluorescent excited states, a nonradiative deexcitation channel is favored. The favored deexcitation channel leads to the short lifetime and low quantum yield of C152 in bulk aqueous environments. 


\section{Reversible C152/DMPC vesicle solution partitioning.}

TCSPC was used to collect C152 fluorescence lifetimes in DMPC vesicle solutions at varying temperatures. The lifetimes and relative amplitudes are shown in Table S1. Data compliment

Figure 6 in showing complete reversibility of C152 partitioning over a broad temperature range.

\begin{tabular}{|c|c|c|c|c|c|c|}
\hline Temp $\left({ }^{\circ} \mathrm{C}\right)$ & $\tau_{1}(\mathrm{~ns})$ & & $\tau_{2}(\mathrm{~ns})$ & 2 & $\tau_{3}(\mathrm{~ns})$ & 3 \\
\hline \multirow{2}{*}{10} & $0.63 \pm$ & $0.62 \pm$ & $2.51 \pm$ & $0.33 \pm$ & $4.89 \pm$ & $0.04 \pm$ \\
& 0.08 & 0.03 & 0.19 & 0.03 & 0.25 & 0.01 \\
\hline \multirow{2}{*}{20} & 0.62 & $0.56 \pm$ & $2.30 \pm$ & $0.41 \pm$ & $4.92 \pm$ & $0.03 \pm$ \\
& \pm 0.11 & 0.03 & 0.12 & 0.03 & 0.19 & 0.01 \\
\hline \multirow{2}{*}{30} & $0.49 \pm$ & $0.30 \pm$ & $1.75 \pm$ & $0.68 \pm$ & $5.01 \pm$ & $0.02 \pm$ \\
& 0.15 & 0.03 & 0.05 & 0.03 & 0.13 & 0.01 \\
\hline \multirow{2}{*}{40} & $0.42 \pm$ & $0.40 \pm$ & $1.29 \pm$ & $0.58 \pm$ & $4.90 \pm$ & $0.02 \pm$ \\
& 0.15 & 0.06 & 0.05 & 0.06 & 0.05 & 0.01 \\
\hline \multirow{2}{*}{50} & $0.41 \pm$ & $0.54 \pm$ & $1.01 \pm$ & $0.45 \pm$ & $4.70 \pm$ & $0.01 \pm$ \\
& 0.14 & 0.09 & 0.04 & 0.09 & 0.07 & 0.01 \\
\hline \multirow{2}{*}{60} & $0.32 \pm$ & $0.61 \pm$ & $0.80 \pm$ & $0.38 \pm$ & $4.42 \pm$ & $0.01 \pm$ \\
& 0.05 & 0.05 & 0.02 & 0.05 & 0.03 & 0.00 \\
\hline \multirow{2}{*}{50} & $0.32 \pm$ & $0.48 \pm$ & $0.94 \pm$ & $0.50 \pm$ & $4.49 \pm$ & $0.02 \pm$ \\
& 0.07 & 0.08 & 0.03 & 0.08 & 0.26 & 0.00 \\
\hline \multirow{2}{*}{40} & $0.44 \pm$ & $0.42 \pm$ & $1.23 \pm$ & $0.56 \pm$ & $4.85 \pm$ & $0.02 \pm$ \\
& 0.08 & 0.03 & 0.02 & 0.03 & 0.04 & 0.00 \\
\hline \multirow{2}{*}{30} & $0.50 \pm$ & $0.34 \pm$ & $1.65 \pm$ & $0.64 \pm$ & $5.08 \pm$ & $0.02 \pm$ \\
& 0.07 & 0.05 & 0.06 & 0.05 & 0.16 & 0.00 \\
\hline \multirow{2}{*}{20} & $0.57 \pm$ & $0.51 \pm$ & $2.18 \pm$ & $0.46 \pm$ & $4.74 \pm$ & $0.03 \pm$ \\
& 0.09 & 0.05 & 0.11 & 0.05 & 0.26 & 0.01 \\
\hline \multirow{2}{*}{10} & $0.63 \pm$ & $0.62 \pm$ & $2.57 \pm$ & $0.34 \pm$ & $5.04 \pm$ & $0.04 \pm$ \\
& 0.10 & 0.04 & 0.24 & 0.04 & 0.34 & 0.01 \\
\hline
\end{tabular}

Table S1. Fluorescence lifetimes of $\mathrm{C} 152$ in DMPC vesicles at $10^{\circ} \mathrm{C}, 20^{\circ} \mathrm{C}, 30^{\circ} \mathrm{C}, 40^{\circ} \mathrm{C}, 50^{\circ} \mathrm{C}$, and $60^{\circ} \mathrm{C}$. The relative amplitudes are corrected for quantum yield. Maximum standard deviations are $\leq 0.2 \mathrm{~ns}$. Note that the QY corrected amplitudes for $10^{\circ}, 30^{\circ}$ and $60^{\circ}$ samples are different than the uncorrected amplitudes that are measured and reported in Table 2 in the manuscript. 